\title{
Adverse intraoperative events during surgical repair of ruptured cerebral aneurysms: a systematic review
}

\author{
William R. Muirhead ${ }^{1}$ (D) Patrick J. Grover ${ }^{1} \cdot$ Ahmed K. Toma $^{1} \cdot$ Danail Stoyanov $^{1,2} \cdot$ Hani J. Marcus $^{1,2} \cdot$ Mary Murphy $^{1}$
}

Received: 4 February 2020 / Revised: 17 April 2020 / Accepted: 29 April 2020 / Published online: 16 June 2020

(C) The Author(s) 2020

\begin{abstract}
Compared with endovascular techniques, clipping of ruptured cerebral aneurysms has been shown to associate with increased morbidity in several studies. Despite this, clipping remains the preferred option for many aneurysms. The objective of this study is to describe the reported adverse events of open repair of ruptured cerebral aneurysms and their impact on patient outcome. The PubMed, Embase and Cochrane databases were searched between June 1999 and June 2019 to identify original studies of at least 100 patients undergoing surgical repair of ruptured cerebral aneurysms and in which adverse event rates were reported. Thirty-six studies reporting adverse events in a total of 12,410 operations for repair of ruptured cerebral aneurysms were included. Surgical adverse events were common with 36 event types reported including intraoperative rupture (median rate of 16.6\%), arterial injury (median rate of 3.8\%) and brain swelling (median rate 5.6\%). Only 6 surgical events were statistically shown to associate with poor outcomes by any author and for intraoperative rupture (the most frequently analysed), there was an even split between authors finding a statistical association with poor outcome and those finding no association. Even with modern surgical techniques, the technical demands of surgical aneurysm repair continue to lead to a high rate of intraoperative adverse events. Despite this, it is not known which of these intraoperative events are the most important contributors to the poor outcomes often seen in these patients. More research directed towards identifying the events that most drive operative morbidity has the potential to improve outcomes for these patients.
\end{abstract}

Keywords Aneurysm clipping $\cdot$ Intraoperative complications $\cdot$ Adverse events $\cdot$ Subarachnoid haemorrhage

\section{Introduction}

Patients with ruptured cerebral aneurysms which are suitable for endovascular repair have been shown to have a reduced risk of death and dependency at 1 year when treated endovascularly compared with open surgical techniques [1,2]. As open surgical repair is associated with a higher rate of aneurysm occlusion in

HJ Marcus and M Murphy are joint senior authors

Electronic supplementary material The online version of this article (https://doi.org/10.1007/s10143-020-01312-4) contains supplementary material, which is available to authorized users.

William R. Muirhead

will.muirhead@doctors.org.uk

1 Department of Neurosurgery, The National Hospital for Neurology and Neurosurgery, Queen Square, London WC1N 3BG, UK

2 Wellcome/EPSRC Centre for Interventional and Surgical Sciences, University College London, London, UK the short and long term, the poor outcomes in the surgical group are believed to be due to the increased morbidity of surgery rather than rebleeding [3]. As many as $43 \%$ of patients undergoing surgery for ruptured cerebral aneurysms will experience an immediate postoperative neurological deterioration [4] and in one series of subarachnoid haemorrhage treated with surgical clipping, $36 \%$ of the deaths and permanent disabilities were attributed to technical intraoperative complications [5]. The profile of adverse events is very different for surgery for unruptured cerebral aneurysms which have much lower morbidity due to more favourable operating conditions [6]. The aim of this review is to better describe the adverse intraoperative events that occur, specifically in surgery for ruptured cerebral aneurysms which appear to cause significant morbidity for this group.

\section{Materials and Methods}

The Preferred Reporting Items for Systematic Reviews and Meta-Analyses (PRISMA) statement was used in the 
preparation of this manuscript [7] and it was registered with the National Institute of Health Research PROSPERO.

\section{Search methods}

The PubMed, Embase and Cochrane databases were searched over a 20-year period between June 1999 and June 2019. The Boolean search term was used ("intracranial aneurysm" OR "intra-cranial aneurysm" OR "cerebral aneurysm" OR "subarachnoid hemorrhage" OR "subarachnoid haemorrhage” OR "SAH”) AND ("surgery" OR "surgical" OR "neurosurgical" OR "neurosurgery" OR "clipping”) AND ("intraoperative" OR "intra-operative") AND ("complications" OR "adverse events"). References were reviewed to identify further articles for inclusion. Two authors (WM and PG) independently identified articles using the above search criteria.

\section{Inclusion and exclusion criteria}

Titles and abstracts were screened to identify publications that met the following inclusion criteria: [1] original studies, [2] reporting a series of at least 100 ruptured aneurysms treated with open surgical repair and [3] describing intraoperative adverse events in these patients.

Papers were excluded if they were: [1] published outside the range June 1999 and June 2019, [2] not in English language and [3] only reported intraoperative adverse events with reference to a larger series of patients (e.g. those undergoing endovascular and open repair).

\section{Data extraction}

The following data were extracted from eligible full articles: [1] year of publication, [2] sample size, [3] whether surgery was performed by a self-reported generalist or self-reported cerebrovascular specialist neurosurgeon, [4] special surgical techniques or adjuncts reported, [5] intraoperative complications reported (the definition of "adverse event" is expanded below), [6] frequency of each complication and [8] impact of complications on patient outcome (if reported).

We follow the CLASSIC authors in their work on surgical complications in taking a broad definition of an "adverse event" as "any deviation from the ideal intraoperative course" [9]. Consequently, the categories of complications recorded include: injuries such as damage to arteries or other structures; incompletion of the surgical goal (in this case invariably failure to secure the aneurysm); increased operative difficulty (due to, for example, brain swelling); interventions that were additional to the ideal intraoperative course (where there is ambiguity as to whether an intervention was considered part of the ideal course by the operating surgeon-e.g. a short period of temporary clip application - we favoured recording the intervention); physiological derangements (e.g. unintentional hypotension or electrolyte imbalance); and neurological harm such as intraoperative strokes or deterioration in conscious level. We formally considered the intraoperative period to begin at induction of anaesthesia and end $2 \mathrm{~h}$ after the patient left the operating theatre; however, frequently adverse events (such as a neurological deficit or a radiological stroke) were recognised some time after this and in such cases, we followed the publication authors in attributing them to the intraoperative period if that was their assessment.

Where authors reported a graded operative difficulty (e.g. slight, moderate, severe brain swelling or easy, moderate or difficult exposure), we recorded this binarily, with "severe" or "difficult" conditions recorded as complications, and considered the "easy", "slight" and "moderate" categories to be within normal limits.

\section{Appraisal of evidence}

The Jadad and Methodological Index for Non-randomised Studies (MINORS) scoring systems were used as frameworks to inform evaluation of the quality of randomised and nonrandomised studies respectively $[10,11]$.

\section{Results}

After automated elimination of duplicates by the systematic review software (Covidence, Melbourne, Australia), our search identified 1309 references for review. After automated screening for duplicates, this was reduced to 1038 references for review. Two further articles were identified from the authors' existing knowledge of the literature for a total of 1040 references for screening (Fig. 1). A total of 943 records were excluded because they did not present original data; reported fewer than 100 open surgical repairs of ruptured intracranial aneurysms; did not report intraoperative complications; were limited to published abstract; or were duplicate references not already screened out by the review software. Ninety-seven articles were identified for full-text review. Full-text screening of these articles led to exclusion of a further 61 references. In all, 36 articles were identified that satisfied the inclusion criteria (Table 1). These 36 articles reported adverse events in 12,410 operations $[4,5,12-21,23-25,27,28,30-35$, 37-49]. Two of these papers [4, 36] both reported the same patient series from the Intraoperative Hypothermia for Aneurysm Surgery Trial-as their focus was on different complication profiles, in these patients, both were included.

In 24 of these 36 articles, patients were identified retrospectively from databases or hospital record. In the remaining 12 patients, they were identified prospectively for the purpose of an either RCT or cohort study (Table 1). 


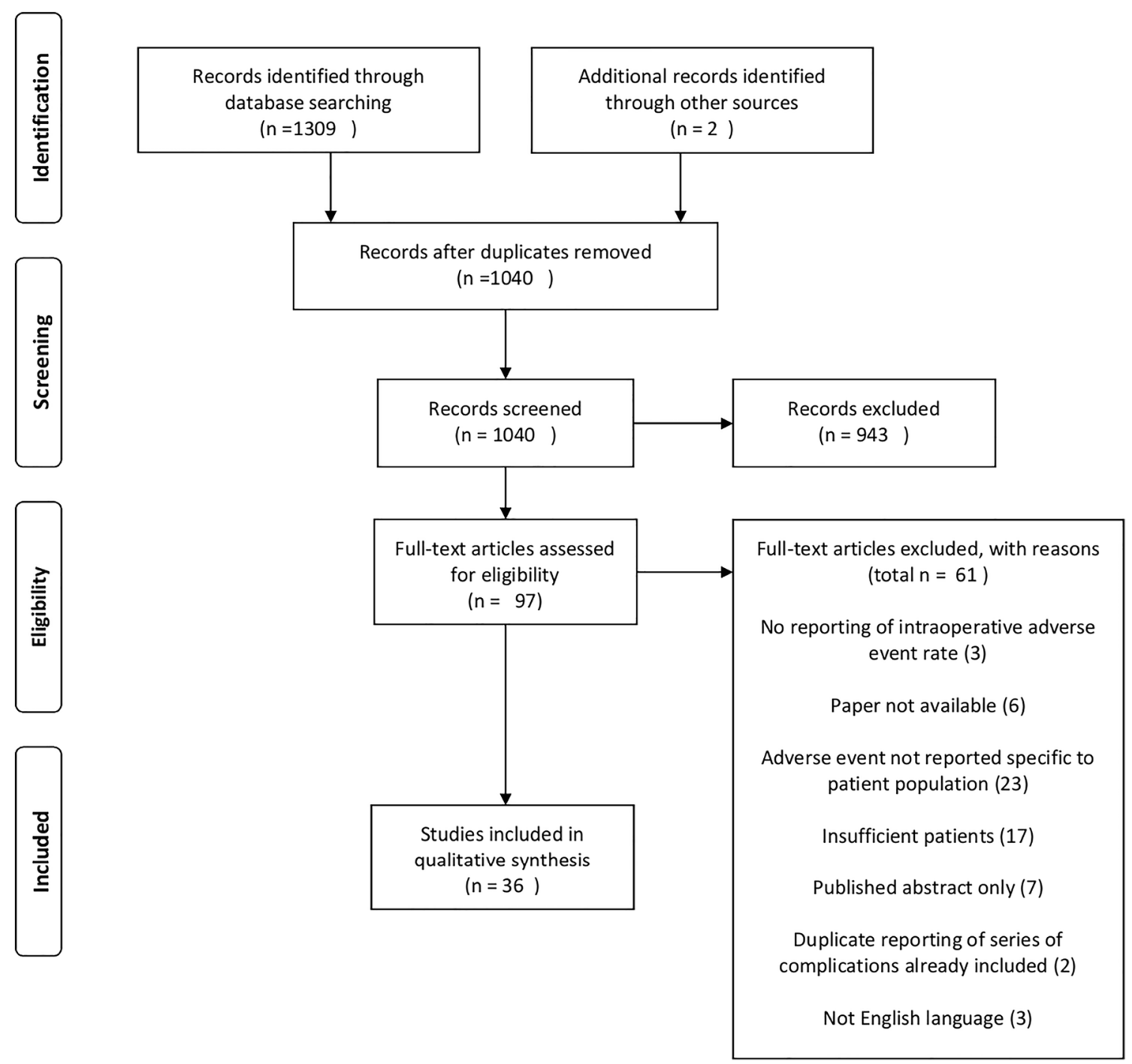

Fig. 1 PRISMA flow diagram

\section{Surgical adverse events}

Surgical adverse events were grouped into arterial and nonarterial injuries, incomplete securing of aneurysms, increased operative difficulty and interventions, and these are presented in Table 2.

By far, most commonly reported adverse event was intraoperative rupture which was reported by 22 of the publications, with a median occurrence rate of $16.6 \%$ (Table 2). A total of 16 authors reported formally significance testing whether rupture was predictive of poor outcome - these were evenly split with $50 \%$ finding an association and the other $50 \%$ finding no association between intraoperative rupture and poor outcome (Fig. 2).

The most commonly reported injuries were to the intracranial arteries with rates varying from 1.1 to $7.1 \%$ with this variation due at least in part to variability in rates of routine check angiography between centres (Table 2). Causes of increased operative difficulty included surgical exposure (35.4\%), swollen brain (5.6\%) and intraoperative rupture $(16.6 \%)$ (Table 2). One author reported an association between challenging operative exposure and poor outcome (Fig. 2).

Intraoperative angiography or Doppler may significantly reduce arterial injury rates as clips were repositioned in as many as $11.9-19 \%$ of cases reported their use. Nonetheless, the rate of arterial injury was as high as $7.1 \%$ in one series when all patients were exposed to control angiography [16]. Intraoperative rupture is the most well-known complication of surgery for ruptured intracranial aneurysms and accordingly the focus of many of the papers we returned, with 25 reporting on rupture. Definitions of rupture are known to vary widely and this is likely to account for the variation in rupture rates (0.6-39.1\%) (Table 2). Several authors presented rupture rates divided by operative stages and we have included those separately. The median rates were $1.1 \%$ during exposure, $15.2 \%$ 
Table 1 Summary of publication included in this review

\begin{tabular}{|c|c|c|c|c|c|c|c|}
\hline Author & Year & Focus of paper & Design & $\begin{array}{l}\text { Number of } \\
\text { operations } \\
\text { for } \\
\text { ruptured } \\
\text { aneurysms }\end{array}$ & $\begin{array}{l}\text { Specialist or } \\
\text { generalist } \\
\text { neurosurgeon }\end{array}$ & $\begin{array}{l}\text { Techniques or } \\
\text { adjuncts }\end{array}$ & $\begin{array}{l}\text { Impact of complications on } \\
\text { outcomes }\end{array}$ \\
\hline Ayling [12] & 2015 & $\begin{array}{l}\text { Review of surgical } \\
\text { complications from the } \\
\text { CONSCIOUS-1 Study into } \\
\text { Clazoneten to prevent surgi- } \\
\text { cal ischaemia }\end{array}$ & $\begin{array}{l}\text { Post hoc } \\
\text { analysis of } \\
\text { RCT } \\
\text { groups }\end{array}$ & 181 & Not reported & None & $\begin{array}{l}\text { Induced hypotension and } \\
\text { intraoperative hypotension } \\
\text { were associated with poor } \\
\text { postoperative GCS }\end{array}$ \\
\hline $\begin{array}{l}\text { Burkhardt } \\
\text { [13] }\end{array}$ & 2016 & $\begin{array}{l}\text { Analysis of preoperative } \\
\text { predictors of intraoperative } \\
\text { rupture }\end{array}$ & $\begin{array}{l}\text { Retrospective } \\
\text { cohort }\end{array}$ & 100 & Not reported & $\begin{array}{l}\text { Temporary } \\
\text { clipping used } \\
\text { variably-no } \\
\text { rate reported }\end{array}$ & $\begin{array}{l}\text { IOR does not have a } \\
\text { significant impact on } \\
\text { clinical outcome }\end{array}$ \\
\hline $\begin{array}{l}\text { Chalouhi } \\
\text { [14] }\end{array}$ & 2012 & $\begin{array}{l}\text { Use of intraoperative } \\
\text { angiography to predict } \\
\text { surgical revision }\end{array}$ & $\begin{array}{l}\text { Retrospective } \\
\text { cohort }\end{array}$ & 360 & Not reported & $\begin{array}{l}\text { Intraoperative } \\
\text { angiography }\end{array}$ & $\begin{array}{l}\text { Ruptured aneurysms were } \\
\text { significantly more likely to } \\
\text { require adjustment } \\
\text { following on-table DSA }\end{array}$ \\
\hline $\begin{array}{l}\text { Darkwah } \\
\text { Oppong } \\
{[15]}\end{array}$ & 2018 & $\begin{array}{l}\text { Factors predicting } \\
\text { intraoperative aneurysm } \\
\text { rupture }\end{array}$ & $\begin{array}{l}\text { Retrospective } \\
\text { cohort }\end{array}$ & 365 & Not reported & None & $\begin{array}{l}\text { IOAR independently } \\
\text { predicted unfavourable } \\
\text { outcome at } 6 \text { months and } \\
\text { in-hospital mortality for pa- } \\
\text { tients with RIA }\end{array}$ \\
\hline Dashti [16] & 2009 & $\begin{array}{l}\text { Assessment of intraoperative } \\
\text { ICG }\end{array}$ & $\begin{array}{l}\text { Prospective } \\
\text { cohort }\end{array}$ & 112 & Not reported & $\begin{array}{l}\text { On-table } \\
\text { angiography } \\
\text { and Doppler }\end{array}$ & No statistical inference drawn \\
\hline $\begin{array}{l}\text { Dhandapani } \\
\text { [17] }\end{array}$ & 2012 & $\begin{array}{l}\text { Assessment of elective } \\
\text { temporary clipping on } \\
\text { neurological outcomes }\end{array}$ & $\begin{array}{l}\text { Prospective } \\
\text { cohort }\end{array}$ & 273 & Not reported & $\begin{array}{l}\text { Mannitol given to } \\
\text { all patients, } \\
\text { elective } \\
\text { temporary } \\
\text { clipping }\end{array}$ & $\begin{array}{l}\text { No operative rupture, short } \\
\text { elective temporary clipping, } \\
\text { rescue temporary clip } \\
\text { (versus elective) was felt to } \\
\text { be protective, total temp } \\
\text { clip time }>20 \text { min predicts } \\
\text { poor outcome }\end{array}$ \\
\hline Doerfler [18] & 2018 & $\begin{array}{l}\text { Incidence and impact of } \\
\text { secondary cerebral insults on } \\
\text { outcome in subarachnoid } \\
\text { haemorrhage }\end{array}$ & $\begin{array}{l}\text { Retrospective } \\
\text { cohort }\end{array}$ & 421 & Not reported & None & $\begin{array}{l}\text { Intraoperative rupture was } \\
\text { associated with } \\
\text { unfavourable outcome }\end{array}$ \\
\hline $\begin{array}{l}\text { Elijovich } \\
\text { [19] }\end{array}$ & 2008 & $\begin{array}{l}\text { Predictors of intraprocedural } \\
\text { rupture in patients treated for } \\
\text { ruptured intracranial } \\
\text { aneurysms }\end{array}$ & $\begin{array}{l}\text { Prospective } \\
\text { cohort }\end{array}$ & 711 & Not reported & None & $\begin{array}{l}\text { Intraoperative rupture higher } \\
\text { risk of poor outcome }(31 \% \\
\text { worse) }\end{array}$ \\
\hline Ferch [20] & 2002 & $\begin{array}{l}\text { Analysis of risk factors for } \\
\text { stroke in surgery for } \\
\text { subarachnoid haemorrhage }\end{array}$ & $\begin{array}{l}\text { Retrospective } \\
\text { cohort }\end{array}$ & 850 & Specialist & None & $\begin{array}{l}\text { Increased incidence of stroke } \\
\text { with prolonged }(>10 \mathrm{~min}) \\
\text { temporary clipping }\end{array}$ \\
\hline $\begin{array}{l}\text { Foroohar } \\
\quad[21]\end{array}$ & 2000 & $\begin{array}{l}\text { Intraoperative variables and } \\
\text { outcome after aneurysm } \\
\text { surgery }\end{array}$ & $\begin{array}{l}\text { Retrospective } \\
\text { cohort }\end{array}$ & 190 & Not reported & None & $\begin{array}{l}\text { Lower maximum } \\
\text { intraoperative systolic } \\
\text { blood pressure was } \\
\text { associated with good } \\
\text { outcome }\end{array}$ \\
\hline $\begin{array}{l}\text { Fridriksson } \\
\text { [5] }\end{array}$ & 2002 & $\begin{array}{l}\text { Prospective collection of } \\
\text { aneurysm complications }\end{array}$ & $\begin{array}{l}\text { Prospective } \\
\text { cohort }\end{array}$ & 355 & Mixed & $\begin{array}{l}\text { Temporary } \\
\text { clipping for } \\
\text { with } \\
\text { neuroprotec- } \\
\text { tion for some } \\
\text { cases }\end{array}$ & $\begin{array}{l}\text { Intraoperative technical } \\
\text { complications caused } 8 \% \text { of } \\
\text { deaths and } 28 \% \text { of } \\
\text { permanent disabilities }\end{array}$ \\
\hline Goertz [22] & 2018 & $\begin{array}{l}\text { Impact of aneurysm shape on } \\
\text { rupture during clipping }\end{array}$ & $\begin{array}{l}\text { Retrospective } \\
\text { cohort }\end{array}$ & 138 & Not reported & None & $\begin{array}{l}\text { Intraoperative rupture had no } \\
\text { impact on rate of } \\
\text { unfavourable outcome }\end{array}$ \\
\hline $\mathrm{Gu}[23]$ & 2018 & $\begin{array}{l}\text { Using cardiac-gated CT angi- } \\
\text { ography to predictive intra- } \\
\text { operative rupture }\end{array}$ & $\begin{array}{l}\text { Prospective } \\
\text { cohort }\end{array}$ & 153 & Specialist & $\begin{array}{r}\text { Cardiac-gated CT } \\
\text { used to predict }\end{array}$ & None \\
\hline
\end{tabular}


Table 1 (continued)

\begin{tabular}{|c|c|c|c|c|c|c|c|}
\hline Author & Year & Focus of paper & Design & $\begin{array}{l}\text { Number of } \\
\text { operations } \\
\text { for } \\
\text { ruptured } \\
\text { aneurysms }\end{array}$ & $\begin{array}{l}\text { Specialist or } \\
\text { generalist } \\
\text { neurosurgeon }\end{array}$ & $\begin{array}{l}\text { Techniques or } \\
\text { adjuncts }\end{array}$ & $\begin{array}{l}\text { Impact of complications on } \\
\text { outcomes }\end{array}$ \\
\hline & & & & & & $\begin{array}{l}\text { intraoperative } \\
\text { rupture }\end{array}$ & \\
\hline Hoff [24] & 2008 & $\begin{array}{l}\text { Impact of intraoperative } \\
\text { hypotension on outcomes }\end{array}$ & $\begin{array}{l}\text { Retrospective } \\
\text { cohort }\end{array}$ & 164 & Not reported & None & $\begin{array}{l}\text { Intraoperative hypotension } \\
\text { was not demonstrated to be } \\
\text { associated with poor } \\
\text { outcome }\end{array}$ \\
\hline Juvela [25] & 2009 & $\begin{array}{l}\text { Whether apolipoprotein } \mathrm{E} \\
\text { genotype predicts outcome } \\
\text { after aneurysmal } \\
\text { subarachnoid haemorrhage }\end{array}$ & $\begin{array}{l}\text { Prospective } \\
\text { cohort }\end{array}$ & 102 & Not reported & $\begin{array}{l}\text { Mannitol in all } \\
\text { operations, } \\
\text { thiopental and } \\
\text { increased BP } \\
\text { prior to } \\
\text { temporary } \\
\text { clipping }\end{array}$ & $\begin{array}{l}\text { Duration of temporary } \\
\text { clipping was associated } \\
\text { with stroke }\end{array}$ \\
\hline $\begin{array}{l}\text { Kapsalaki } \\
\text { [26] }\end{array}$ & 2008 & $\begin{array}{l}\text { The role of intraoperative } \\
\text { micro-Doppler ultrasound in } \\
\text { verifying proper clip place- } \\
\text { ment in intracranial aneu- } \\
\text { rysm surgery }\end{array}$ & $\begin{array}{l}\text { Retrospective } \\
\text { cohort }\end{array}$ & 121 & Not reported & None & No comment on outcome \\
\hline $\begin{array}{l}\text { Kashkoush } \\
\quad[27]\end{array}$ & 2017 & $\begin{array}{l}\text { Utility of SSEP in predicting } \\
\text { stroke }\end{array}$ & $\begin{array}{l}\text { Retrospective } \\
\text { cohort }\end{array}$ & 133 & Not reported & SSEP monitoring & SSEP is predictive of stroke \\
\hline Kivisaari [28] & 2004 & $\begin{array}{l}\text { Investigation of utility of } \\
\text { control angiography }\end{array}$ & $\begin{array}{l}\text { Prospective } \\
\text { cohort }\end{array}$ & 493 & Experienced & None & $\begin{array}{l}\text { No correlation with } \\
\text { neurological outcome } \\
\text { reported }\end{array}$ \\
\hline $\begin{array}{l}\text { Lakicevic } \\
\text { [29] }\end{array}$ & 2015 & $\begin{array}{l}\text { Impact of intraoperative } \\
\text { rupture on outcome }\end{array}$ & $\begin{array}{l}\text { Retrospective } \\
\text { cohort }\end{array}$ & 536 & Not reported & None & $\begin{array}{l}\text { Intraoperative rupture } \\
\text { increases rate of } \\
\text { postoperative deficit }\end{array}$ \\
\hline Leipzig [30] & 2005 & $\begin{array}{l}\text { Rupture rates of aneurysm } \\
\text { surgery }\end{array}$ & $\begin{array}{l}\text { Retrospective } \\
\text { cohort }\end{array}$ & 970 & Not reported & None & $\begin{array}{l}\text { IOR appeared to increase risk } \\
\text { of stroke or death (although } \\
\text { not statistical focus of } \\
\text { paper) }\end{array}$ \\
\hline Le Roux [31] & 2001 & $\begin{array}{l}\text { Review of blood transfusion in } \\
\text { aneurysm surgery }\end{array}$ & $\begin{array}{l}\text { Retrospective } \\
\text { cohort }\end{array}$ & 441 & Not reported & None & None \\
\hline $\operatorname{Lin}[32]$ & 2013 & $\begin{array}{l}\text { Factors associated with poor } \\
\text { outcome in patients with } \\
\text { major intraoperative rupture } \\
\text { of cerebral aneurysms }\end{array}$ & $\begin{array}{l}\text { Prospective } \\
\text { cohort }\end{array}$ & 647 & Not reported & None & $\begin{array}{l}\text { Intraoperative rupture is a risk } \\
\text { factor for poor outcome }\end{array}$ \\
\hline $\begin{array}{l}\text { Luostarinen } \\
\quad[33]\end{array}$ & 2015 & $\begin{array}{l}\text { Frequency of transfusion } \\
\text { during aneurysm surgery }\end{array}$ & $\begin{array}{l}\text { Retrospective } \\
\text { cohort }\end{array}$ & 488 & Not reported & None & $\begin{array}{l}\text { Intraoperative RBC } \\
\text { transfusion associated with } \\
\text { poor neurological outcome }\end{array}$ \\
\hline Mahaney [4] & 2012 & $\begin{array}{l}\text { Predictors of postoperative } \\
\text { deterioration }\end{array}$ & $\begin{array}{l}\text { Retrospective } \\
\text { analysis of } \\
\text { RCT }\end{array}$ & 1000 & Not reported & None & $\begin{array}{l}\text { Logistic regression model - } \\
\text { Intentional intraoperative } \\
\text { hypotension, blood loss, } \\
\text { duration of temporary clip } \\
\text { application } \geq 20 \text { min, diffi- } \\
\text { culty of aneurysm exposure } \\
\text { were found to associate } \\
\text { with poor outcomes }\end{array}$ \\
\hline $\begin{array}{l}\text { McLaughlin } \\
\text { [34] }\end{array}$ & 2004 & $\begin{array}{l}\text { Analysis of early } \\
\text { surgery-related complica- } \\
\text { tions }\end{array}$ & $\begin{array}{l}\text { Retrospective } \\
\quad \text { single } \\
\text { surgeon } \\
\text { series }\end{array}$ & 179 & $\begin{array}{l}80 \% \\
\quad \text { specialist }\end{array}$ & None & $\begin{array}{l}\text { Surgical complications were } \\
\text { associated with worse GOS } \\
\text { at } 3 \text { months }\end{array}$ \\
\hline $\begin{array}{l}\text { Molyneux } \\
\text { [35] }\end{array}$ & 2002 & Trial of clipping versus coiling & $\mathrm{RCT}$ & 1004 & Generalist & None & $\begin{array}{l}\text { No relationship between } \\
\text { intraoperative } \\
\text { complications and outcome }\end{array}$ \\
\hline Nguyen [36] & 2010 & $\begin{array}{l}\text { Effect of perioperative } \\
\text { hypothermia on occurrence }\end{array}$ & $\mathrm{RCT}$ & 1000 & Not reported & Hypothermia & $\begin{array}{l}\text { Hypothermia was not } \\
\text { associated with increased }\end{array}$ \\
\hline
\end{tabular}


Table 1 (continued)

\begin{tabular}{|c|c|c|c|c|c|c|c|}
\hline Author & Year & Focus of paper & Design & $\begin{array}{l}\text { Number of } \\
\text { operations } \\
\text { for } \\
\text { ruptured } \\
\text { aneurysms }\end{array}$ & $\begin{array}{l}\text { Specialist or } \\
\text { generalist } \\
\text { neurosurgeon }\end{array}$ & $\begin{array}{l}\text { Techniques or } \\
\text { adjuncts }\end{array}$ & $\begin{array}{l}\text { Impact of complications on } \\
\text { outcomes }\end{array}$ \\
\hline & & $\begin{array}{l}\text { of cardiovascular events in } \\
\text { patients undergoing cerebral } \\
\text { aneurysm surgery (also from } \\
\text { IHAST) }\end{array}$ & & & & & $\begin{array}{l}\text { occurrence of any single } \\
\text { cardiovascular event or } \\
\text { composite cardiovascular } \\
\text { event }\end{array}$ \\
\hline Park [37] & 2016 & $\begin{array}{l}\text { Risk factors for intraoperative } \\
\text { rupture of MCA aneurysms }\end{array}$ & $\begin{array}{l}\text { Retrospective } \\
\text { cohort }\end{array}$ & 182 & Specialist & Mannitol & $\begin{array}{l}\text { No statistical inference drawn } \\
\text { about outcome }\end{array}$ \\
\hline $\begin{array}{l}\text { Sandalcioglu } \\
\text { [38] }\end{array}$ & 2004 & $\begin{array}{l}\text { Effect of intraoperative rupture } \\
\text { on outcome }\end{array}$ & $\begin{array}{l}\text { Retrospective } \\
\text { cohort }\end{array}$ & 169 & Not reported & $\begin{array}{l}\text { Rupture } \\
\text { controlled with } \\
\text { double suction, } \\
\text { temporary } \\
\text { clipping and/or } \\
\text { focal } \\
\text { tamponade }\end{array}$ & $\begin{array}{l}\text { Rupture has a trend to increase } \\
\text { morbidity and mortality } \\
\text { when IAR occurs in } \\
\text { patients with poor initial } \\
\text { condition although this was } \\
\text { not statistically significant }\end{array}$ \\
\hline Sheth [39] & 2014 & $\begin{array}{l}\text { Effect of intraoperative rupture } \\
\text { on vasospasm }\end{array}$ & $\begin{array}{l}\text { Retrospective } \\
\text { cohort }\end{array}$ & 500 & Not reported & None & $\begin{array}{l}\text { Intraoperative rupture was not } \\
\text { associated with subsequent } \\
\text { vasospasm }\end{array}$ \\
\hline $\begin{array}{l}\text { Umredkar } \\
\text { [40] }\end{array}$ & 2010 & $\begin{array}{l}\text { Incidence of intracerebral } \\
\text { infarcts after aneurysm } \\
\text { clipping }\end{array}$ & $\begin{array}{l}\text { Prospective } \\
\text { cohort }\end{array}$ & 174 & Not reported & None & $\begin{array}{l}\text { Longer temporary clipping } \\
\text { associated with infarct }\end{array}$ \\
\hline $\begin{array}{l}\text { Van Lindert } \\
\text { [41] }\end{array}$ & 2001 & $\begin{array}{l}\text { The influence of surgical } \\
\text { experience on the rate of } \\
\text { intraoperative aneurysm } \\
\text { rupture }\end{array}$ & $\begin{array}{l}\text { Retrospective } \\
\text { cohort }\end{array}$ & 308 & $\begin{array}{l}40 \% \text { by } \\
\text { surgeons } \\
\text { clipping }> \\
\text { 10/year }\end{array}$ & None & $\begin{array}{l}\text { IAR rate higher for } \\
\text { non-specialist surgeons }\end{array}$ \\
\hline Wester [42] & 2009 & $\begin{array}{l}\text { Single surgeon complications } \\
\text { from aneurysm surgery }\end{array}$ & $\begin{array}{l}\text { Retrospective } \\
\text { single } \\
\text { surgeon } \\
\text { series }\end{array}$ & 223 & Generalist & None & $\begin{array}{l}\text { Intraoperative ruptures } \\
\text { decreased with increasing } \\
\text { surgical experience }\end{array}$ \\
\hline $\begin{array}{l}\text { Yamamoto } \\
{[43]}\end{array}$ & 2017 & $\begin{array}{l}\text { Effect of perforator infarction } \\
\text { after ACOM clipping }\end{array}$ & $\begin{array}{l}\text { Retrospective } \\
\text { cohort }\end{array}$ & 104 & Not reported & $\begin{array}{l}\text { Temporary } \\
\text { clipping, } \\
\text { Doppler, ICG }\end{array}$ & $\begin{array}{l}\text { Intraoperative rupture and } \\
\text { temporary clipping were } \\
\text { associated with perforator } \\
\text { infarction, perforator } \\
\text { infarction associated with } \\
\text { poor neurological outcome }\end{array}$ \\
\hline Yee [44] & 2017 & $\begin{array}{l}\text { Tranfusion rates in intracranial } \\
\text { aneurysm surgery }\end{array}$ & $\begin{array}{l}\text { Retrospective } \\
\text { cohort }\end{array}$ & 141 & Not reported & None & No comment on outcome \\
\hline Zhang [45] & 2012 & $\begin{array}{l}\text { Impact of clipping versus } \\
\text { coiling in over } 60\end{array}$ & $\begin{array}{l}\text { Retrospective } \\
\text { cohort }\end{array}$ & 122 & Not reported & None & $\begin{array}{l}\text { No statistical relationship } \\
\text { between complications and } \\
\text { outcome }\end{array}$ \\
\hline
\end{tabular}

during aneurysm dissection and $6.7 \%$ during clip application or manipulation (Table 2).

Temporary clipping was the most commonly reported additional surgical intervention and was used as often as $76.2 \%$ of the time in one series [17]. Temporary clipping was used both pre-emptively to soften aneurysms for dissection and reactively to control haemorrhage. Length of temporary clipping was found by many authors to be associated with poor outcome and we present separately the duration of clipping by one author reporting a large series where $27.9 \%$ of patients underwent temporary clipping for $<10 \mathrm{~min}, 10.4 \%$ for $10-20$ min and $5.8 \%$ for over $20 \mathrm{~min}$. Three papers found a statistical association between prolonged temporary clipping and poor outcome (Fig. 2), although one tested this and found no association.

\section{Systemic adverse events}

Physiological derangements and interventions that would ordinarily lie outside of the neurosurgical field are presented together in Table 3. 
Table 2 Surgical events

\begin{tabular}{|c|c|c|c|c|c|}
\hline Type & Specific complication & $\begin{array}{l}\text { Number of papers } \\
\text { reporting }\end{array}$ & $\begin{array}{l}\text { Median complication } \\
\text { rate }\end{array}$ & Min & Max \\
\hline \multirow[t]{8}{*}{ Arterial injury } & Arterial injury/occlusion (any) & 7 & $3.8 \%$ & $1.1 \%$ & $7.1 \%$ \\
\hline & Arterial injury/occlusion (not further specified) & 4 & $4.6 \%$ & $1.1 \%$ & $7.1 \%$ \\
\hline & Arterial occlusion - large vessel & 3 & $2.9 \%$ & $2.7 \%$ & $6.1 \%$ \\
\hline & Arterial occlusion - perforator & 1 & $3.8 \%$ & $3.8 \%$ & $3.8 \%$ \\
\hline & Arterial stenosis & 1 & $0.6 \%$ & $0.6 \%$ & $0.6 \%$ \\
\hline & Distal embolus & 1 & $0.6 \%$ & $0.6 \%$ & $0.6 \%$ \\
\hline & Major haemorrhage & 1 & $6.3 \%$ & $6.3 \%$ & $6.3 \%$ \\
\hline & $\begin{array}{l}\text { Suboptimal clip placement recognised by } \\
\text { angiography/Doppler }\end{array}$ & 2 & $15.5 \%$ & $11.9 \%$ & $19.0 \%$ \\
\hline \multirow{3}{*}{$\begin{array}{l}\text { Incomplete securing of } \\
\text { aneurysm }\end{array}$} & Partial occlusion with neck remnant & 3 & $5.4 \%$ & $3.9 \%$ & $10.5 \%$ \\
\hline & $\begin{array}{l}\text { Partial occlusion with incompletely secure rupture } \\
\text { point }\end{array}$ & 2 & $3.1 \%$ & $2.8 \%$ & $3.4 \%$ \\
\hline & Surgery abandoned & 1 & $0.8 \%$ & $0.8 \%$ & $0.8 \%$ \\
\hline \multirow{8}{*}{$\begin{array}{l}\text { Increased operative } \\
\text { difficulty }\end{array}$} & Difficult operative exposure & 1 & $35.3 \%$ & $35.3 \%$ & $35.3 \%$ \\
\hline & Intraoperative rupture (any stage) & 22 & $16.6 \%$ & $0.6 \%$ & $39.1 \%$ \\
\hline & Intraoperative rupture (during exposure) & 4 & $1.1 \%$ & $0.4 \%$ & $5.0 \%$ \\
\hline & Intraoperative rupture (during aneurysm dissection) & 4 & $18.6 \%$ & $2.9 \%$ & $24.0 \%$ \\
\hline & $\begin{array}{l}\text { Intraoperative rupture (during clip application or } \\
\text { manipulation) }\end{array}$ & 4 & $6.7 \%$ & $0.9 \%$ & $9.4 \%$ \\
\hline & Parent vessel vasospasm & 1 & $9.1 \%$ & $9.1 \%$ & $9.1 \%$ \\
\hline & Swollen brain & 3 & $5.6 \%$ & $2.8 \%$ & $8.5 \%$ \\
\hline & Technical failure (non-release of aneurysm clip) & 1 & $0.4 \%$ & $0.4 \%$ & $0.4 \%$ \\
\hline \multirow[t]{11}{*}{ Intervention } & Administration of local vasodilators & 1 & $1.1 \%$ & $1.1 \%$ & $1.1 \%$ \\
\hline & Cerebral angiogram (intraop or within $2 \mathrm{~h}$ ) & 1 & $9.2 \%$ & $9.2 \%$ & $9.2 \%$ \\
\hline & Further neurosurgery (including bypass) & 3 & $0.6 \%$ & $0.5 \%$ & $0.6 \%$ \\
\hline & ICP monitor & 1 & $2.9 \%$ & $2.9 \%$ & $2.9 \%$ \\
\hline & Lumbar drain & 1 & $31.9 \%$ & $31.9 \%$ & $31.9 \%$ \\
\hline & Temporary clipping (any) & 9 & $44.5 \%$ & $13.2 \%$ & $76.2 \%$ \\
\hline & Temporary clipping $<10 \mathrm{~min}$ & 1 & $27.9 \%$ & $27.9 \%$ & $27.9 \%$ \\
\hline & Temporary clipping $>20 \mathrm{~min}$ & 1 & $5.8 \%$ & $5.8 \%$ & $5.8 \%$ \\
\hline & Temporary clipping 10-20 min & 1 & $10.4 \%$ & $10.4 \%$ & $10.4 \%$ \\
\hline & Temporary clipping of ACA & 1 & $21.6 \%$ & $21.6 \%$ & $21.6 \%$ \\
\hline & Temporary clipping of ICA & 1 & $8.9 \%$ & $8.9 \%$ & $8.9 \%$ \\
\hline \multirow[t]{6}{*}{ Non-arterial injury } & Cranial nerve injury & 1 & $2.2 \%$ & $2.2 \%$ & $2.2 \%$ \\
\hline & CSF leak & 1 & $1.1 \%$ & $1.1 \%$ & $1.1 \%$ \\
\hline & Derangement of neuromonitoring & 1 & $24.1 \%$ & $24.1 \%$ & $24.1 \%$ \\
\hline & Direct trauma to brain parenchyma & 2 & $0.6 \%$ & $0.6 \%$ & $0.6 \%$ \\
\hline & Eye injury & 1 & $0.6 \%$ & $0.6 \%$ & $0.6 \%$ \\
\hline & New subarachnoid haemorrhage or ICH & 2 & $1.0 \%$ & $0.4 \%$ & $1.6 \%$ \\
\hline
\end{tabular}

The most commonly reported systemic adverse events were red cell transfusion (17.7\%) and unplanned administration of neuroprotective drugs (15.5\%) (Table 3) although, notably, one series reported a very high rate of induced hypotension $(44.6 \%)$ [50] - presumably as a strategy to mitigate the effects of intraoperative rupture.

Intraoperative hypertension, induced hypotension and transfusion were all reported to associate with poor outcome (Fig. 2).

\section{Neurological adverse events}

Neurological harm occurred commonly. Radiologically confirmed infarcts, clinical strokes and undefined neurological deteriorations were all reported and presented in Table 4. In one paper that reported all-cause postoperative neurological deterioration, this occurred in $42.6 \%$ of patients [4]. The median rate of radiological stroke was $31.7 \%$, which was significantly higher than the rate of clinical stroke (8.8\%) (Table 4). 
Fig. 2 Intraoperative events found to be statistically associated with poor outcome

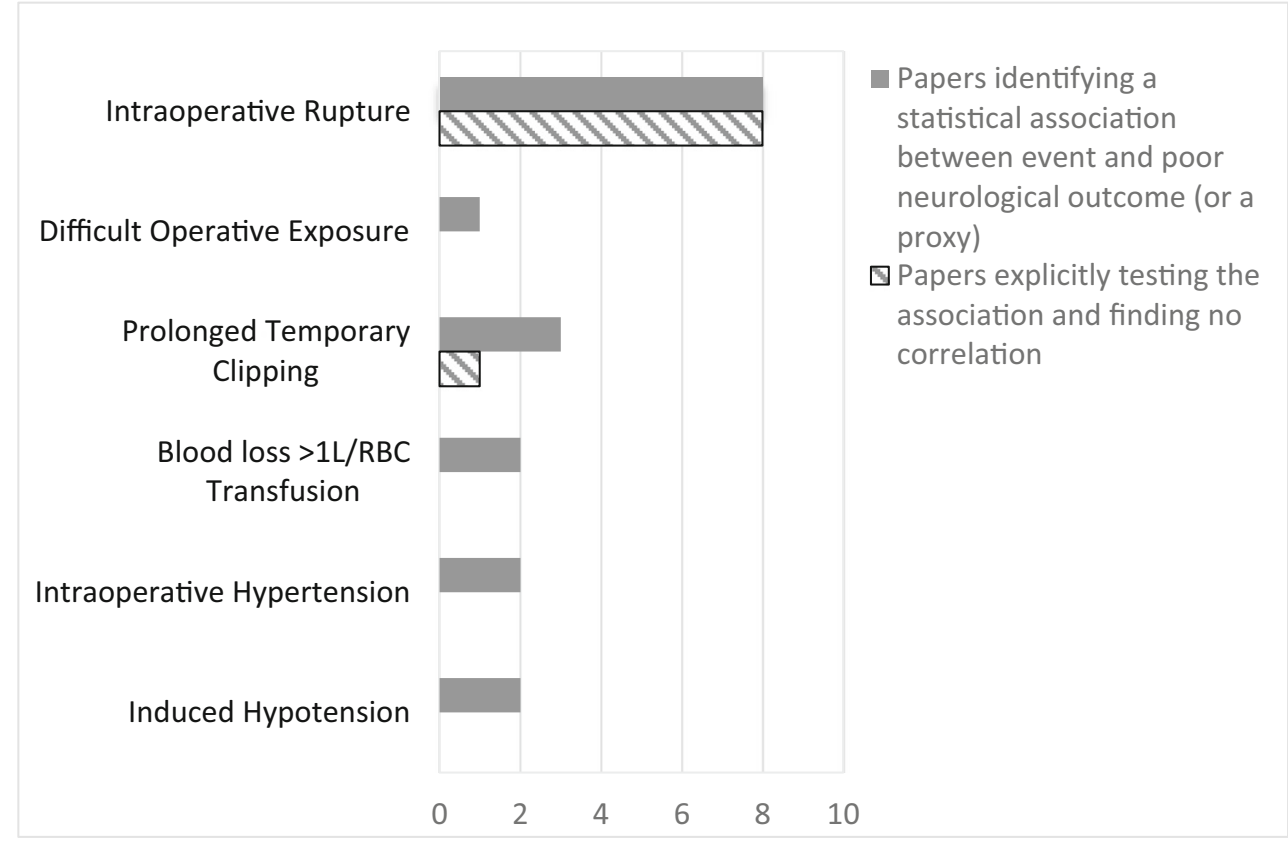

\section{Discussion}

\section{Principle findings}

Surgery for ruptured intracranial aneurysms is associated with a wide range of adverse events as demonstrated by the range of modalities reported by the authors included in this review.

The adverse surgical conditions encountered by neurovascular surgeons, which include difficult surgical exposure $(35.4 \%)$, swollen brain $(5.6 \%)$ and intraoperative rupture $(16.6 \%)$, all increase the operative difficulty of the procedure (Table 2). It is likely that all of these contribute to the high rate of arterial injury (4.6\%), direct parenchymal injury $(0.6 \%)$ and prolonged temporary ischaemic time (> $10 \mathrm{~min}$ in $16.2 \%$ of patients) (Table 2 ). These in turn contribute to the high rate of new postoperative radiological infarction $(31.7 \%)$ seen in these patients (Table 4$)$. Despite the high rate of intraoperative adverse events, only rupture, difficult exposure and prolonged temporary clipping were found to associate with poor outcome by any of the authors in our series (Fig. 2).

Table 3 Systemic events

\begin{tabular}{|c|c|c|c|c|c|}
\hline Type & Specific complication & $\begin{array}{l}\text { Number of papers } \\
\text { reporting }\end{array}$ & $\begin{array}{l}\text { Median } \\
\text { complication } \\
\text { rate }\end{array}$ & Min & Max \\
\hline \multirow[t]{6}{*}{ Intervention } & $\begin{array}{l}\text { Non neurosurgical procedure (retrograde jugular venous catheter, } \\
\text { reintubation, cardioversion) }\end{array}$ & 2 & $1.1 \%$ & $0.6 \%$ & $1.6 \%$ \\
\hline & Tranfusion of FFP & 2 & $2.0 \%$ & $0.9 \%$ & $3.1 \%$ \\
\hline & Tranfusion of platelets & 1 & $1.2 \%$ & $1.2 \%$ & $1.2 \%$ \\
\hline & Transfusion of RBC & 3 & $17.7 \%$ & $7.6 \%$ & $27.2 \%$ \\
\hline & Unplanned administration of mannitol & 1 & $1.7 \%$ & $1.7 \%$ & $1.7 \%$ \\
\hline & Unplanned administration of neuroprotective drugs & 1 & $15.5 \%$ & $15.5 \%$ & $15.5 \%$ \\
\hline \multirow{7}{*}{$\begin{array}{l}\text { Physiological } \\
\text { derangement }\end{array}$} & Anaemia & 1 & $3.8 \%$ & $3.8 \%$ & $3.8 \%$ \\
\hline & Cardiac event or instability & 2 & $3.9 \%$ & $0.6 \%$ & $7.3 \%$ \\
\hline & Electrolyte or glucose disturbance & 1 & $1.3 \%$ & $1.3 \%$ & $1.3 \%$ \\
\hline & Hypertension (intended) & 1 & $2.5 \%$ & $2.5 \%$ & $2.5 \%$ \\
\hline & Hypertension (unintended) & 2 & $4.1 \%$ & $2.8 \%$ & $5.5 \%$ \\
\hline & Hypotension (intended) & 3 & $4.6 \%$ & $3.3 \%$ & $44.6 \%$ \\
\hline & Hypotension (unintended) & 2 & $4.3 \%$ & $3.7 \%$ & $5.0 \%$ \\
\hline
\end{tabular}


Table 4 Neurological events

\begin{tabular}{|c|c|c|c|c|c|}
\hline Type & Specific complication & Number of papers reporting & Median complication rate & Min & Max \\
\hline \multirow[t]{6}{*}{ Neurological harm } & Clinical stroke (immediate) & 2 & $8.8 \%$ & $7.9 \%$ & $9.8 \%$ \\
\hline & Death & 1 & $0.4 \%$ & $0.4 \%$ & $0.4 \%$ \\
\hline & Postoperative neurological deterioration (any cause) & 1 & $42.6 \%$ & $42.6 \%$ & $42.6 \%$ \\
\hline & Radiological stroke (any) & 3 & $31.7 \%$ & $4.4 \%$ & $32.4 \%$ \\
\hline & Radiological cortical stroke & 1 & $15.4 \%$ & $15.4 \%$ & $15.4 \%$ \\
\hline & Radiological perforator stroke & 1 & $23.1 \%$ & $23.1 \%$ & $23.1 \%$ \\
\hline
\end{tabular}

On-table Doppler, indocyanine green (ICG) angiography and digital formal angiography were all used to mitigate vascular injury and confirm aneurysm occlusion. Whilst ICG angiography is now regarded as the gold standard in many units, it was only reported to be used universally by one author [43]. This may be a reflection the fact that many of the series reported in this paper are already relatively historic or simply that the authors of these papers did not feel it was important to explicitly mention in their papers.

Intraoperative rupture was the most commonly reported adverse event but there were discordant results when statistical testing was applied regarding whether it affected neurological outcome (Fig. 2). Whilst a rupture during the final stages of aneurysm dissection when proximal control has been obtained, it is certain that an early rupture with bleeding that is difficult to control or requires prolonged ischaemic time with the application of temporary clips will worsen outcome and this is likely reflected in the associations that were identified between induced hypotension, prolonged temporary clipping and transfusion. Higher rupture rates were associated with less experienced surgeons [41, 42]. Despite it being the most commonly reported adverse event, the only adjunct widely reported to manage intraoperative rupture was the use of temporary clipping in these larger series. Prolonged temporary clipping was associated with poor neurological outcomes, and neuroprotective measures and hypothermia were tried by several authors to mitigate this [4, 5]. Microdialysis has previously demonstrated that a fall of brain tissue $\mathrm{PO}_{2}$ to less than $8 \mathrm{mmHg}$ for longer than $30 \mathrm{~min}$ in any arterial territory is strongly predictive of cerebral infarction [51]. Further techniques for managing intraoperative rupture that were not described in these series include cardiac standstill with adenosine [52] or rapid ventricular pacing [53]. Whilst it is possible that experienced neurosurgeons are able to mitigate the impact of rupture [13] reducing its impact on patient outcome, predissection ruptures or ruptures that extend into the aneurysm neck are particularly hard to manage and almost certainly associated with a poor prognosis [42, 54]. Many authors advocate the use of endoscopic approaches to better visualize vessel branches and reduce the rate of inadvertent injury [55,
56] but the endoscope offers no advantage over the microscope for control of intraoperative rupture.

\section{Comparison with other studies}

Comparing the intraoperative adverse events in our review with those of the aneurysm series published before, our 2decade period of capture yields a mixed message of progress. Whilst intraoperative rupture remains commonly reported, its effect on outcome is contentious, and certainly none of the modern studies in our review reported the 3-fold increased rate of unfavourable outcome reported by Batjer and Samson [57]. Conversely, the rates of arterial injury are if anything higher than historical series - Sundt [58] for example reported a major vessel injury rate of $0.62 \%$ and perforator injury in $1.4 \%$ of cases (versus $2.9 \%$ and $3.8 \%$ respectively in this review (Table 2)), although it is likely that an increased use of postoperative imaging is leading to the higher rates in our series. The principle surgical strategy for managing rupture (application of temporary clips) has remained popular over this time $[57,59]$.

It is worth also drawing comparison with the single other large review in the literature covering complications of cerebral aneurysm surgery which bears detailed comparison with this one. Wong et al. [60] review complications reported by in 19 papers covering open cerebrovascular surgery with a total of 7562 patients in total principally undergoing surgery for ruptured or unruptured aneurysms. They identify five of the same intraoperative events as our review-stroke, intraoperative rupture, incomplete aneurysm obliteration, major vessel occlusion, failure to secure rupture site, and the rates of these are similar to those we found - although our review reports a further 45 types of event. This difference is due to the following three factors: (i) we include approximately twice as many patients across the papers we identified, (ii) the focus of this review is more targeted (looking just at intraoperative complications in ruptured aneurysm surgery) resulting in a more granular reporting (e.g. separate rates for perforator and cortical stroke, where this is rolled up into a single complication in the other review) and (iii) we use a wider definition of adverse 
event meaning that rates of adverse conditions, such as brain swelling as well as interventions such as temporary clipping, are included in our review but excluded from Wong et al.

\section{Limitations}

It would be important to know whether the rate of adverse events in aneurysm surgery has decreased over the 20-year period of our literature review. Modern techniques, such as on-table indocyanine green angiography as well as the increasing concentration of vascular cases in the hands of specialist neurovascular surgeons, may be expected to have had a significant effect on adverse event rates. Unfortunately, the papers we have collected do not lend themselves to a meaningful analysis of this question due to differences in reporting standards, frequently many years of data capture within each paper and confounders, e.g. single author series where we might expect a reduction in event rates over time with greater operator experience [5,34].

Analysis of the clinically relevant complications that occur during clipping is made particularly challenging by the heterogenous patient group: the ultimate impact of cerebral insult on patient outcome is difficult to correlate when the outcome itself is difficult to predict. To further confound this, the overwhelming majority of events were only reported by a few authors. With the exception of rupture (22 authors), temporary clipping ( 9 authors) and arterial injury ( 7 authors), the other event rates (e.g. brain swelling, parent vessel vasospasm or cranial nerve injury) were all reported by fewer than 5 authors.

Similarly due to the heterogeneity of the date reported in these papers, the data did not lend themselves to analysis of particular patient subgroups which may have been significant, e.g. we might have expected higher rates of brain swelling rates in patients with associated haematomas with mass effect and lower rates of retraction injury in patients with middle cerebral artery aneurysms due to their relative accessibility.

There are a wide range of adverse event rates reported in the literature and this often reflects differences in definition (e.g. many authors reported rupture as an adverse event only if it occurred in a pre-dissection phase or before proximal control could be obtained, whereas others reported rupture at any stage). For the interventions, in particular, surgeon preference played a large part-with some surgeons choosing to use temporary clips almost routinely as a softening strategy even for uncomplicated aneurysms - whilst others typically deployed them only as an emergency measure to gain vascular control. These differences place a natural limit on the utility of reporting adverse events simply as the raw rate.

As our search strategy returned papers that focused on "adverse events" or "complications", this study, interventions which may not have been regarded as meeting the definition of an adverse event by the study author are likely to be underemphasised. Notably, frequency of intraoperative external ventricular drain placement (which presumably occurred commonly) was not reported in any of our series - we hypothesise that this is because most authors would not regard it as an adverse event - although it would have met the wider definition we use in this paper. Similarly, cerebrospinal fluid (CSF) diversion by lumbar drain insertion rates was only commented on in one paper at $31.9 \%$ (Table 2). As with temporary clipping, an episode of intraoperative CSF diversion can be indicative of an adverse event resulting in a deviation of surgery from its operative course, or simply a surgeon's routine practice for the particular case in hand. Being unable to distinguish between these situations was a significant limitation of the approach we have taken in this paper.

Moreover, whilst challenging aneurysm morphology and severe brain swelling are widely believed to significantly increase the risks associated with aneurysm surgery, these were only rarely reported by authors in our review. It seems likely that events that exist on a spectrum (such as degree of brain swelling) and which are consequently more difficult to report may have been relatively under-reported.

\section{Summary}

Whilst endovascular techniques have become increasingly used in open aneurysm surgery, open surgical repair remains the preferred modality for many aneurysms [2, 3]. Compared with the fast pace of innovation seen in endovascular techniques for coiling, only a small number of new technologies have been adopted in open aneurysm surgery since Yasargil developed the microscopic approaches used by the majority of surgeons today [61] There is considerable morbidity that is associated with open repair of cerebral aneurysms attributable to technical surgical challenges. The overwhelmingly most commonly reported surgical adverse event is intraoperative rupture and it is certain that an uncontrolled rupture (occurring either during pre-dissection or from a tear in the neck of the aneurysm) leads to poor outcomes for many patients. Despite this, the papers in this review were split when trying to show whether there was a statistical association between rupture and poor outcome. This underscores the point that beyond surgeons' experience and intuitions, we do not have an evidence-based understanding of the drivers of morbidity in aneurysm surgery. No papers, for example, demonstrated an association between brain swelling and poor patient outcome, despite the surgical intuition that this increase in the difficulty of the surgical conditions is highly likely to impact upon the morbidity of the surgery. Further research is needed into this topic and would help by a consensus on the definitions of each intraoperative events to enable comparison between patient series. A better understanding of the relationship between the adverse 
events of aneurysm clipping and their contribution to patient morbidity would not only help target further research to address these problems but also help surgeons mitigate the impact of those events when they occur.

Funding information HJM is supported by the Wellcome/EPSRC Centre for Interventional and Surgical Sciences (WEISS) and NIHR BRC Neuro-oncology theme.

\section{Compliance with ethical standards}

Conflict of interest None

Ethical approval Not applicable

Informed consent Not applicable

Open Access This article is licensed under a Creative Commons Attribution 4.0 International License, which permits use, sharing, adaptation, distribution and reproduction in any medium or format, as long as you give appropriate credit to the original author(s) and the source, provide a link to the Creative Commons licence, and indicate if changes were made. The images or other third party material in this article are included in the article's Creative Commons licence, unless indicated otherwise in a credit line to the material. If material is not included in the article's Creative Commons licence and your intended use is not permitted by statutory regulation or exceeds the permitted use, you will need to obtain permission directly from the copyright holder. To view a copy of this licence, visit http://creativecommons.org/licenses/by/4.0/.

\section{References}

1. Molyneux AJ, Kerr RSC, Yu L-M, Clarke M, Sneade M, Yarnold JA, Sandercock P (2005) International Subarachnoid Aneurysm Trial (ISAT) of neurosurgical clipping versus endovascular coiling in 2143 patients with ruptured intracranial aneurysms: a randomised comparison of effects on survival, dependency, seizures, rebleeding, subgroups, and. Lancet 366(9488):809-817. https:// doi.org/10.1016/S0140-6736(05)67214-5

2. McDougall CG, Spetzler RF, Zabramski JM et al (2012) The barrow ruptured aneurysm trial: clinical article. J Neurosurg 116(1): 135-144. https://doi.org/10.3171/2011.8.JNS101767

3. Rodríguez-Hernández A, Sughrue ME, Akhavan S, HabdankKolaczkowski J, Lawton MT (2013) Current management of middle cerebral artery aneurysms: surgical results with a clip first policy. Neurosurgery 72(3):415-427. https://doi.org/10.1227/NEU. 0b013e3182804aa2

4. Mahaney KB, Todd MM, Bayman EO, Torner JC (2012) Acute postoperative neurological deterioration associated with surgery for ruptured intracranial aneurysm: incidence, predictors, and outcomes. J Neurosurg 116(6):1267-1278. https://doi.org/10.3171/ 2012.1.JNS111277

5. Fridriksson S, Säveland H, Jakobsson K-EK-E et al (2002) Intraoperative complications in aneurysm surgery: a prospective national study. J Neurosurg 96(3):515-522. https://doi.org/10. 3171/jns.2002.96.3.0515

6. Ogilvy CS, Jordan NJ, Ascanio LC, Enriquez-Marulanda AA, Salem MM, Moore JM, Thomas AJ (2019) Surgical and endovascular comprehensive treatment outcomes of unruptured intracranial aneurysms: reduction of treatment bias. World Neurosurg 126:e878-e887. https://doi.org/10.1016/j.wneu.2019. 03.005

7. Liberati A, Altman DG, Tetzlaff J, Mulrow C, Gøtzsche PC, Ioannidis JPA, Clarke M, Devereaux PJ, Kleijnen J, Moher D (2009) The PRISMA statement for reporting systematic reviews and meta-analyses of studies that evaluate health care interventions: explanation and elaboration. J Clin Epidemiol 62(10):e1-e34. https://doi.org/10.1016/j.jclinepi.2009.06.006

8. S Hammond J, Muirhead W, Zaitoun AM, Cameron IC, Lobo DN (2012) Comparison of liver parenchymal ablation and tissue necrosis in a cadaveric bovine model using the Harmonic Scalpel, the LigaSure, the Cavitron Ultrasonic Surgical Aspirator and the Aquamantys devices. HPB (Oxford) 14(12):828-832. https://doi. org/10.1111/j.1477-2574.2012.00547.x

9. Rosenthal R, Hoffmann H, Clavien PA, Bucher HC, Dell-Kuster S (2015) Definition and classification of intraoperative complications (classic): Delphi study and pilot evaluation. World J Surg 39(7): 1663-1671. https://doi.org/10.1007/s00268-015-3003-y

10. Jadad AR, Moore RA, Carroll D, Jenkinson C, Reynolds DJM, Gavaghan DJ, McQuay HJ (1996) Assessing the quality of reports of randomized clinical trials: is blinding necessary? Control Clin Trials 17(1):1-12

11. Slim K, Nini E, Forestier D, Kwiatkowski F, Panis Y, Chipponi J (2003) Methodological index for non-randomized studies (minors): development and validation of a new instrument. ANZ J Surg 73(9):712-716

12. Ayling OG, Ibrahim GM, Drake B, Torner JC, Macdonald RL (2015) Operative complications and differences in outcome after clipping and coiling of ruptured intracranial aneurysms. J Neurosurg 123(3 CC-Stroke):621-628. https://doi.org/10.3171/ 2014.11.JNS141607

13. Burkhardt JK, Neidert MC, Mohme M, Seifert B, Regli L, Bozinov O (2015) Initial clinical status and spot sign are associated with intraoperative aneurysm rupture in patients undergoing surgical clipping for aneurysmal subarachnoid hemorrhage. J Neurol Surg A Cent Eur Neurosurg 77(2):130-138. https://doi.org/10.1055/s0035-1558414

14. Chalouhi N, Theofanis T, Jabbour P, Dumont AS, Gonzalez LF, Starke RM, Dalyai RT, Hann S, Rosenwasser R, Tjoumakaris S (2012) Safety and efficacy of intraoperative angiography in craniotomies for cerebral aneurysms and arteriovenous malformations: a review of 1093 consecutive cases. Neurosurgery 71(6):E550-E551. https://doi.org/10.1227/NEU.0b013e318271ebfc

15. Darkwah Oppong M, Pierscianek D, Ahmadipour Y, Dinger TF, Dammann P, Wrede KH, Özkan N, Müller O, Sure U, Jabbarli R (2018) Intraoperative aneurysm rupture during microsurgical clipping: risk re-evaluation in the post-international subarachnoid aneurysm trial era. World Neurosurg 119:e349-e356. https://doi.org/ 10.1016/j.wneu.2018.07.158

16. Dashti R, Laakso A, Niemelä M, Porras M, Hernesniemi J (2009) Microscope-integrated near-infrared indocyanine green videoangiography during surgery of intracranial aneurysms: the Helsinki experience. Surg Neurol 71(5):543-550. https://doi.org/ 10.1016/j.surneu.2009.01.027

17. Dhandapani S, Pal SS, Gupta SK, Mohindra S, Chhabra R, Malhotra SK (2013) Does the impact of elective temporary clipping on intraoperative rupture really influence neurological outcome after surgery for ruptured anterior circulation aneurysms? - A prospective multivariate study. Acta Neurochir (Wien) 155(2):237246. https://doi.org/10.1007/s00701-012-1571-2

18. Doerfler S, Faerber J, McKhann GM et al (2018) The incidence and impact of secondary cerebral insults on outcome after aneurysmal subarachnoid hemorrhage. World Neurosurg 114:e483-e494. https://doi.org/10.1016/j.wneu.2018.02.195 
19. Elijovich L, Higashida RT, Lawton MT, Duckwiler G, Giannotta S, Johnston SC (2008) Predictors and outcomes of intraprocedural rupture in patients treated for ruptured intracranial aneurysms: the CARAT study. Stroke 39(5):1501-1506. https://doi.org/10.1161/ STROKEAHA.107.504670

20. Ferch R, Pasqualin A, Pinna G, Chioffi F, Bricolo A (2009) Temporary arterial occlusion in the repair of ruptured intracranial aneurysms: an analysis of risk factors for stroke. J Neurosurg 97(4): 836-842. https://doi.org/10.3171/jns.2002.97.4.0836

21. Foroohar M, Macdonald L, Roth S, Stoodley M, Ph D, Weir B (2000) Intraoperative variables and early outcome after aneurysm surgery. Surg Neurol 54:304-315

22. Goertz L, Kasuya H, Hamisch C et al (2018) Impact of aneurysm shape on morbidity after clipping of unruptured intracranial aneurysms. Acta Neurochir (Wien) 160(11):2169-2176. https://doi.org/ 10.1007/s00701-018-3675-9

23. Gu Y, Xu L, Hu C, Luo M, Zhang H, Liu X (2018) Monitoring dynamic morphological changes with electrocardiography-gated dynamic 4-dimensional computed tomography angiography to predict intraoperative rupture of intracranial aneurysms. J Comput Assist Tomogr 42(2):286-292. https://doi.org/10.1097/RCT. 0000000000000671

24. Hoff RG, Van Dijk GW, Mettes S et al (2008) Hypotension in anaesthetized patients during aneurysm clipping: not as bad as expected? Acta Anaesthesiol Scand 52(7):1006-1011. https://doi.org/ 10.1111/j.1399-6576.2008.01682.x

25. Juvela S, Siironen J, Lappalainen J (2009) Apolipoprotein E genotype and outcome after aneurysmal subarachnoid hemorrhage: clinical article. J Neurosurg 110(5):989-995. https://doi.org/10.3171/ 2008.11.JNS081266

26. Kapsalaki EZ, Lee GP, Robinson JS 3rd, Grigorian AA, Fountas KN (2008) The role of intraoperative micro-Doppler ultrasound in verifying proper clip placement in intracranial aneurysm surgery. $\mathrm{J}$ Clin Neurosci Off J Neurosurg Soc Australas 15(2):153-157. https://doi.org/10.1016/j.jocn.2006.11.006

27. Kashkoush AI, Jankowitz BT, Nguyen C et al (2017) Perioperative stroke after cerebral aneurysm clipping: risk factors and postoperative impact. J Clin Neurosci 44:188-195. https://doi.org/10.1016/j. jocn.2017.06.030

28. Kivisaari RP, Porras M, Öhman J, Siironen J, Ishii K, Hernesniemi J (2004) Routine cerebral angiography after surgery for saccular aneurysms: is it worth it? Neurosurgery 55(5):1015-1022. https:// doi.org/10.1227/01.NEU.0000141043.07303.60

29. Lakicevic N, Vujotic L, Radulovic L, Cvrkota L (2015) Factors influencing intraoperative rupture of intracranial aneurysms. Turk Neurosurg 25(6):858-885. https://doi.org/10.5137/1019-5149. JTN.12966-14.2

30. Leipzig TJ, Morgan J, Horner TG, Payner T, Redelman K, Johnson CS (2005) Analysis of intraoperative rupture in the surgical treatment of 1694 saccular aneurysms. Neurosurgery 56(3):455-466. https://doi.org/10.1227/01.NEU.0000154697.75300.C2

31. Le Roux PD, Elliott JP, Winn HR (2001) Blood transfusion during aneurysm surgery. Neurosurgery 49(5):1068-1075. http://ovidsp. ovid.com/ovidweb.cgi? T=JS\&PAGE $=$ reference $\& \mathrm{D}=$ emed7\&NEWS=N\&AN=32995843. Accessed 15 Jun 2020

32. Lin T-KK, Hsieh T-CC, Tsai H-CC LY-JJ, Lin C-LL, Huang Y-CC (2013) Factors associated with poor outcome in patients with major intraoperative rupture of intracranial aneurysm. Acta Neurol Taiwan 22(3):106-111

33. Luostarinen T, Lehto H, Skrifvars MB, Kivisaari R, Niemelä M, Hernesniemi J, Randell T, Niemi T (2015) Transfusion frequency of red blood cells, fresh frozen plasma, and platelets during ruptured cerebral aneurysm surgery. World Neurosurg 84(2):446-450. https://doi.org/10.1016/j.wneu.2015.03.053

34. Mclaughlin N, Bojanowski MW (2004) Early surgery-related complications after aneurysm clip placement: an analysis of causes and patient outcomes. J Neurosurg 101(4):600-606. https://doi.org/10. 3171/jns.2004.101.4.0600

35. Molyneux AJ (2002) International Subarachnoid Aneurysm Trial (ISAT) of neurosurgical clipping versus endovascular coiling in 2143 patients were ruptured intracranial aneurysms: a randomised trial. Lancet 360:1267-1274

36. Nguyen HP, Zaroff JG, Bayman EO, Gelb AW, Todd MM, Hindman BJ (2010) Perioperative hypothermia (33 degrees C) does not increase the occurrence of cardiovascular events in patients undergoing cerebral aneurysm surgery: findings from the Intraoperative Hypothermia for Aneurysm Surgery Trial. Anesthesiology 113(2):327-342. https://doi.org/10.1097/ALN. 0b013e3181dfd4f7

37. Park J, Son W, Park K-S, Kang D-H, Shin IH (2016) Intraoperative premature rupture of middle cerebral artery aneurysms: risk factors and sphenoid ridge proximation sign. J Neurosurg 125(5):12351241. https://doi.org/10.3171/2015.10.JNS151586

38. Sandalcioglu IE, Schoch B, Regel JP, Wanke I, Gasser T, Forsting M, Stolke D, Wiedemayer H (2004) Does intraoperative aneurysm rupture influence outcome? Analysis of 169 patients. Clin Neurol Neurosurg 106(2):88-92. https://doi.org/10.1016/j.clineuro.2003. 10.011

39. Sheth SA, Hausrath D, Numis AL, Lawton MT, Josephson SA (2013) Intraoperative rerupture during surgical treatment of aneurysmal subarachnoid hemorrhage is not associated with an increased risk of vasospasm. J Neurosurg 120(2):409-414. https:// doi.org/10.3171/2013.10.jns13934

40. Umredkar A, Gupta SK, Khandelwal N, Chhabra R, Mathuriya SN, Pathak A, Tiwari MK, Mukherjee KK, Mohindra S, Singla N, Salunke P (2010) Intracerebral infarcts following clipping of intracranial aneurysms: Incidence, clinical correlation and outcome. $\mathrm{Br} \mathrm{J}$ Neurosurg 24(2):156-162. https://doi.org/10.3109/ 02688690903513412

41. Van Lindert EJ, BöcherSchwarz HG, Perneczky A (2001) The influence of surgical experience on the rate of intraoperative aneurysm rupture and its impact on aneurysm treatment outcome. Surg Neurol 56(3):151-156. https://doi.org/10.1016/S0090-3019(01) 00547-X

42. Wester K (2009) Lessons learned by personal failures in aneurysm surgery: what went wrong, and why? Acta Neurochir (Wien) 151(9):1013-1024. https://doi.org/10.1007/s00701-009-0452-9

43. Yamamoto Y, Fukuda H, Yamada D et al (2017) Association of perforator infarction with clinical courses and outcomes following surgical clipping of ruptured anterior communicating artery aneurysms. World Neurosurg 107:2-5. https://doi.org/10.1016/j.wneu. 2017.08.086

44. Yee JN, Koht A, McCarthy RJ, Bebawy JF (2017) Factors associated with blood transfusion during intracranial aneurysm surgery. $\mathrm{J}$ Clin Anesth 36:164-167. https://doi.org/10.1016/j.jclinane.2016. 10.031

45. Zhang QR, Zhang X, Wu Q, Shi JX, Wang HD, Hang CH, Cheng HL, Liu JM (2012) The impact of microsurgical clipping and endovascular coiling on the outcome of cerebral aneurysms in patients over 60 years of age. J Clin Neurosci 19(8):1115-1118. https://doi.org/10.1016/j.jocn.2011.11.017

46. Goertz L, Hamisch C, Telentschak S, Kabbasch C, von Spreckelsen N, Stavrinou P, Timmer M, Goldbrunner R, Brinker G, Krischek B (2018) Impact of aneurysm shape on intraoperative rupture during clipping of ruptured intracranial aneurysms. World Neurosurg 118: e806-e812. https://doi.org/10.1016/j.wneu.2018.07.058

47. Nguyen H, Zaroff J, Bayman EO, Gelb A, Todd M, Hindman B (2010) Perioperative hypothermia $\left(33{ }^{\circ} \mathrm{C}\right.$ ) does not increase the occurrence of cardiovascular events in patients undergoing cerebral aneurysm surgery. Anesthesiology 2:327-342

48. Siasios I, Kapsalaki EZ, Fountas KN (2012) The role of intraoperative micro-Doppler ultrasound in verifying proper clip placement 
in intracranial aneurysm surgery. Neuroradiology 54(10):11091118. https://doi.org/10.1007/s00234-012-1023-y

49. Lakićević N, Prstojević B, Rasulić L, Vujotić L, Vukašinović Ivan, Miličić B, Savić A, Živković B, Rotim K, Samardžić M (2015) Intraoperative aneurysmal rupture: clinical outcome following open surgery or endovascular treatment. Acta Clin Croat 54(3):285-293

50. Mahaney KB, Todd MM, Torner JC (2011) Variation of patient characteristics, management, and outcome with timing of surgery for aneurysmal subarachnoid hemorrhage. J Neurosurg 114(4 CCStroke):1045-1053. https://doi.org/10.3171/2010.11.JNS10795

51. Kett-White R, Hutchinson PJ, Al-Rawi PG et al (2002) Cerebral oxygen and microdialysis monitoring during aneurysm surgery: effects of blood pressure, cerebrospinal fluid drainage, and temporary clipping on infarction. J Neurosurg 96(6):1013-1019. https:// doi.org/10.3171/jns.2002.96.6.1013

52. Guinn NR, McDonagh DL, Borel CO et al (2011) Adenosineinduced transient asystole for intracranial aneurysm surgery: a retrospective review. J Neurosurg Anesthesiol 23(1):35-40. https:// doi.org/10.1097/ANA.0b013e3181ef2b11

53. Saldien V, Menovsky T, Rommens T et al (2012) Rapid ventricular pacing for flow arrest during cerebrovascular surgery: revival of an old concept. Neurosurgery 70(2 Suppl Operative):270-275. http:// ovidsp.ovid.com/ovidweb.cgi?T $=\mathrm{JS} \& \mathrm{PAGE}=$ reference $\& \mathrm{D}=$ emed13\&NEWS $=\mathrm{N} \& A N=366383888$. Accessed 15 Jun 2020

54. Zhen Y, Yan K, Zhang H, Zhao S, Xu Y, Zhang H, He L, Shen L (2014) Analysis of the relationship between different bleeding positions on intraoperative rupture anterior circulation aneurysm and surgical treatment outcome. Acta Neurochir (Wien) 156(3):481491. https://doi.org/10.1007/s00701-013-1953-0
55. Reisch R, Fischer R, Stadie A, Kockro R, Cesnulis E (2014) The supraorbital endoscopic approach for aneurysms. World Neurosurg 82(6):S130-S137. https://doi.org/10.1016/j.wneu.2014.07.038

56. Fischer G, Stadie A, Reisch R et al (2011) The keyhole concept in aneurysm surgery: results of the past 20 years. Neurosurgery 68(SUPPL. 1). https://doi.org/10.1227/NEU.0b013e31820934ca

57. Batjer H, Samson D (1986) Intraoperative aneurysmal rupture: incidence, outcome, and suggestions for surgical management. Neurosurgery 18(6):701-707. https://doi.org/10.1227/00006123198606000-00004

58. Sundt TM, Kobayashi S, Fode NC, Whisnant JP (1982) Results and complications of surgical management of 809 intracranial aneurysms in 722 cases. J Neurosurg 56(6):753-765. https://doi.org/ $10.3171 /$ jns.1982.56.6.0753

59. Drake CG (1979) The treatment of aneurysms of the posterior circulation. Clin Neurosurg 26:96-144 http://ci.nii.ac.jp/naid/ 10004782537/en/. Accessed August 7, 2019

60. Wong JM, Ziewacz JE, Ho AL, Panchmatia JR, Kim AH, Bader AM, Thompson BG, du R, Gawande AA (2012) Patterns in neurosurgical adverse events: open cerebrovascular neurosurgery. Neurosurg Focus 33(5):E15. https://doi.org/10.3171/2012.7. focus 12181

61. Maurice-Williams RS (2004) Aneurysm surgery after the International Subarachnoid Aneurysm Trial (ISAT). J Neurol Neurosurg Psychiatry 75(6):807-808. https://doi.org/10.1136/ jnnp.2004.036962

Publisher's note Springer Nature remains neutral with regard to jurisdictional claims in published maps and institutional affiliations. 